\title{
Endothelial cell adhesion molecules in meningococcal disease
}

\author{
P B Baines, O Marzouk, A P J Thomson, J A Sills, F A I Riordan, C A Hart
}

\begin{abstract}
Background-Endothelial damage is important in meningococcal disease. Cell adhesion molecules, including $\mathbf{P}$ selectin, $E$ selectin, and intercellular cell adhesion molecule 1 (ICAM-1), are expressed by activated endothelium and then subsequently shed.
\end{abstract}

Methods-ICAM-1, P selectin, and E selectin were measured on admission to hospital in children with meningococcal infections.

Results-Concentrations of shed cell adhesion molecules are reported for 78 children. Eleven did not have meningococcal disease. Of the 67 with meningococcal disease, 40 had mild disease (Glasgow meningococcal septicaemia prognostic score (GMSPS) $<8$ ) and 27 had severe disease (GMSPS $\geqslant 8$ ). $E$ selectin and ICAM-1 values were higher in those with meningococcal disease. The $\mathrm{E}$ selectin values in those with severe disease were higher than in those with mild disease. $P$ selectin concentrations were not altered in meningococcal disease, but those who died had lower concentrations.

Conclusions-Endothelial activation in meningococcal disease is reflected by shed ICAM-1 and E selectin concentrations. (Arch Dis Child 1999;80:74-76)

Royal Liverpool

Children's Hospital,

Eaton Road, Liverpool

L12 2AP, UK

P B Baines

Institute of Child

Health, Royal

Liverpool Children's

Hospital

O Marzouk

A P J Thomson

J A Sills

Department of

Paediatrics,

Birmingham

Heartlands Hospital, Birmingham B9 5ST, UK

F A I Riordan

Department of Medical Microbiology and Genitourinary Medicine, Liverpool

University, Liverpool L69 3GA, UK

C A Hart

Correspondence to: Dr Baines.

email: p.baines@

liverpool.ac.uk

Accepted 21 July 1998 Keywords: meningococcal disease; intercellular cell adhesion molecule 1; P selectin; E selectin

Endothelial cell activation by inflammatory cytokines alters endothelial cell function. This, and the subsequent leucocyte-endothelial interaction, ${ }^{1}$ is of fundamental importance in the pathology of inflammation and healing, and so also in the changes of sepsis.

In invasive meningococcal disease, high serum concentrations of lipooligosaccharide $(\mathrm{LOS})^{2}$ are produced, which correlate well with disease severity. LOS causes a host response, which includes the release of cytokines, again well related to disease severity. ${ }^{34}$ Cytokines, including tumour necrosis factor (TNF) and interleukin 1 (IL-1), activate endothelial cells, altering function in several ways. Presynthesised P selectin, which is stored in the WeibelPalade bodies of endothelial cells (and the $\alpha$ granules of platelets) is released on to the endothelial cell surface on activation. It subsequently disappears from the cell surface and at least a proportion is released into the bloodstream, where it may be detected. ${ }^{5}$ Intercellular cell adhesion molecule 1 (ICAM-1), a member of the immunoglobulin family of adhesion molecules, and E selectin are expressed constitutively on the surface of the endothelium. On activation, the expression of both increases on the cell surface, although being dependent on protein synthesis, this is slower and more sustained than $\mathrm{P}$ selectin. Again, a proportion is released into the circulation. ${ }^{6-9}$ Measurement of shed cell adhesion molecules in blood is feasible in humans, whereas direct immunohistochemical demonstration of the cell adhesion molecules on the endothelial cell surface is more difficult.

Binding of the leucocytes to endothelial cells might cause dysfunction of the endothelial cells directly. ${ }^{10}$

Evaluation of the role of cell adhesion molecules will offer a better understanding of the pathophysiology of the cardiovascular collapse in sepsis, and might allow the development of new strategies to manage sepsis. ${ }^{11}{ }^{12}$ However, as abnormalities of neutrophilleucocyte interaction cause the recurrent infections in leucocyte adhesion deficiencies, and ICAM-1 knockout mice have a lower mortality from Haemophilus influenzae infection, but a higher mortality from Streptococcus pneumoniae infections, ${ }^{13}$ any proposed treatment must be evaluated carefully. Treatments, including the choice of fluid used in resuscitation ${ }^{14}$ or the inotrope used, ${ }^{15}$ affect the expression of cell adhesion molecules, and so may unwittingly affect endothelial cell responses.

A rise in concentrations of ICAM-1, E selectin, and vascular cell adhesion molecule (VCAM-1) have been described in adults with meningococcal sepsis or meningitis, ${ }^{16}{ }^{17}$ but $\mathrm{P}$ selectin concentrations were unchanged. There are no data from children.

We sought to characterise the endothelial dysfunction of meningococcal disease by measuring the circulating serum concentrations of $\mathrm{P}$ selectin, E selectin, and ICAM-1 on admission to hospital in a large group of children with meningococcal disease.

\section{Methods}

Children admitted to hospital with a clinical diagnosis of meningococcal disease were inease in Merseyside, which ran from November 1988 until August 1990 and from September 1992 until April 1994. Children admitted to the Royal Liverpool Children's Hospital, Arrowe Park, Whiston, Warrington and the Countess of Chester Hospitals were enrolled.

The study was approved by the local ethics committee and consent was obtained from the children's parents.

Children were scored using the Glasgow meningococcal septicaemia prognostic score cluded from two studies of meningococcal dis- 


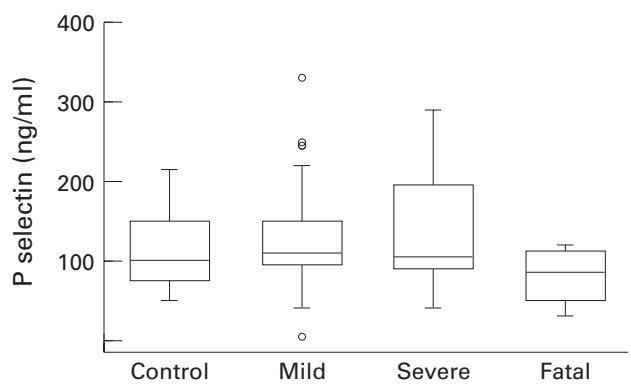

Figure 1 Shed P selectin concentration in controls $(n=11)$ and children with meningococcal disease stratified by GMSPS into mild disease $(n=40)$ and severe disease $(n=19)$, and in eight fatal cases. The box represents the interquartile range, with the central line being the median. The whiskers represent the adjacent values. Values outside the whiskers are plotted individually.

(GMSPS), ${ }^{18}$ a score of 8 or more identifies a group with mortality of $30 \% .^{19}$

Blood was obtained on admission to hospital. Serum was separated and stored at $-70^{\circ} \mathrm{C}$ until analysed. E selectin, P selectin, and ICAM-1 were measured in duplicate by a commercially available immunoenzymometric technique ( R + D Systems Europe, Abingdon, UK). Cytokines were measured as described previously. ${ }^{20}$

Statistical analysis was performed using STATA (Stata Corporation, Texas, USA), using the two sample Wilcoxon rank sum test and Spearman correlation coefficient.

\section{Results}

Blood was obtained on admission from 78 children. In 11 children, the final diagnosis was not meningococcal disease (controls), and in 67 children the final diagnosis was of meningococcal disease. Of the 67 children with meningococcal disease, 40 had mild disease (GMSPS $<8$ ) and 27 had severe disease (GMSPS $\geqslant 8$ ). Of the 27 with severe disease, eight children died. All children with GMSPS $<8$ survived.

In 11 children, a diagnosis of meningococcal infection was considered on admission, although the final diagnosis was not meningococcal disease. The children ranged in age from 1 to 180 months. The median age was 11 months. The diagnoses included viral infections (six), chest infections (two), non-meningococcal bacterial infection (one), haemophilus meningitis (one), and febrile fit (one). All of these children survived. These are the non-meningococcal infectious disease controls.

Of the 67 children with meningococcal disease, 39 were boys and 28 were girls. Of the eight children who died, two were girls. The ages of the children ranged from 4 to 170 months, with a median of 24 months. This was similar for each of the three groups: mild disease, severe disease, and fatal cases.

$P$ selectin values on admission were similar in the controls and the groups with mild and severe disease (fig 1). The serum concentration of shed P selectin was lower in those who subsequently died. The median concentration of those who subsequently died $(85 \mathrm{ng} / \mathrm{ml})$ was significantly lower than those who survived with meningococcal disease $(110 \mathrm{ng} / \mathrm{ml}$; $\mathrm{p}=0.04$ by Wilcoxon rank sum test).
The $\mathrm{P}$ selectin concentrations were related to the platelet count on admission, with higher $\mathrm{P}$ selectin values seen with higher platelet counts (Spearman correlation coefficient 0.32; $\mathrm{p}=0.01)$. The children with more severe disease had lower platelet counts and counts were lowest in those who subsequently died.

The serum concentrations of shed E selectin were higher in the children with meningococcal disease than in the controls (median, $315 \mathrm{ng}$ / $\mathrm{ml} v 100 \mathrm{ng} / \mathrm{ml}$, respectively; $\mathrm{p}=0.0001$ by Wilcoxon rank sum test) (fig 2). The E selectin concentrations in the severely affected children (GMSPS $\geqslant 8$ ) were higher than in those who were less severely affected (median, $275 \mathrm{ng} / \mathrm{ml}$ v $360 \mathrm{ng} / \mathrm{ml}$, respectively; $\mathrm{p}=0.01$ by Wilcoxon rank sum test). The serum shed E selectin concentrations in those who died were lower than in those with severe disease who survived (median, $300 \mathrm{ng} / \mathrm{ml}$ v $392 \mathrm{ng} / \mathrm{ml}$, respectively; $\mathrm{p}=0.04$ by Wilcoxon rank sum).

The serum concentrations of shed ICAM-1 were higher in those with meningococcal disease than in controls (median, $380 \mathrm{ng} / \mathrm{ml} v$ $500 \mathrm{ng} / \mathrm{ml}$, respectively; $\mathrm{p}=0.0001$ by Wilcoxon rank sum test) (fig 3). There was no significant difference between those with mild and severe disease.

The concentrations of shed E selectin and ICAM-1 were related, higher concentrations of E selectin being seen in those patients with higher levels of ICAM-1 (Spearman correlation coefficient, $0.7 ; \mathrm{p}=0.001)$. There was no

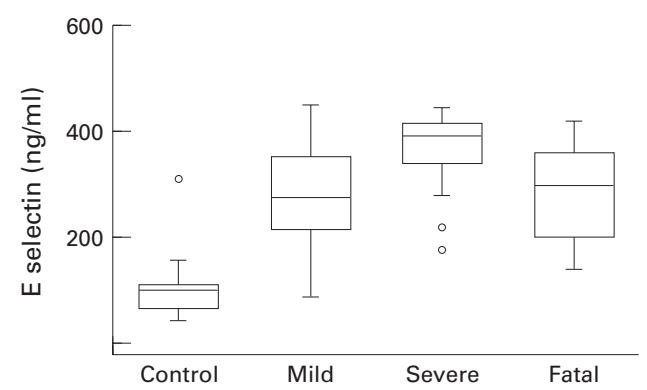

Figure 2 Shed E selectin concentrations in controls $(n=11)$ and children with meningococcal disease stratified by GMSPS into mild disease $(n=40)$ and severe disease $(n=19)$, and in eight fatal cases. The box represents the interquartile range, with the central line being the median. The whiskers represent the adjacent values. Values outside the whiskers are plotted individually.

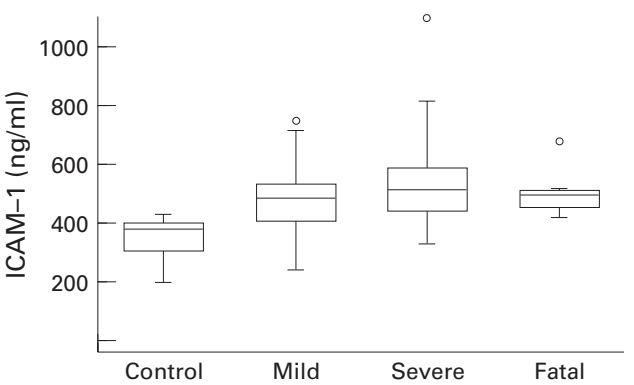

Figure 3 Shed ICAM-1 concentrations in controls $(n=11)$ and children with meningococcal disease stratified by GMSPS into mild disease $(n=40)$ and severe disease $(n=19)$, and in eight fatal cases. The box represents the interquartile range, with the central line being the median. The whiskers represent the adjacent values. Values outside the whiskers are plotted individually. 
relation between $\mathrm{P}$ selectin and ICAM-1 or E selectin.

Apart from a relation between $\mathrm{P}$ selectin and IL-10 (Spearman correlation coefficient, -0.38 ; $\mathrm{p}=0.03$ ) there was no significant relation between the concentrations of shed cell adhesion molecules and serum concentrations of LOS, TNF, IL-6, or IL-1.

\section{Discussion}

We have demonstrated differences in concentrations of shed cell adhesion molecules in children with meningococcal disease. Both ICAM-1 and E selectin were increased in children with meningococcal disease and concentrations of $\mathrm{E}$ selectin were higher still in those with severe disease. ICAM-1 and E selectin concentrations were related to one another. ICAM-1 and E selectin are both dependent on protein synthesis for increased synthesis and share similar time courses, which may explain their relation. Surprisingly, in children with severe meningococcal disease, the $\mathrm{E}$ selectin concentrations in those children who subsequently died were less than in those who survived. This contrasts with previous work in critically ill adults, where Cowley et al reported the highest concentrations of $\mathrm{E}$ selectin in those who subsequently died, although they did find that not all of those who died had high concentrations of $\mathrm{E}$ selectin. ${ }^{7}$

The ICAM-1 concentrations in children with meningococcal disease were higher than in the controls. There was no significant difference in ICAM-1 concentrations between those with mild and severe meningococcal disease, nor between those who survived and those who died. Previous work ${ }^{78}$ has shown a rise of up to fourfold in ICAM-1 levels in critically ill adults with sepsis, which was highest in those who subsequently died.

$\mathrm{P}$ selectin was not raised in children with meningococcal disease and, more particularly, was low in those with meningococcal disease who died. This is consistent with $\mathrm{P}$ selectin concentrations in adults with meningococcal disease. ${ }^{16}$ This contrasts with the results of Sakamaki and et $a l,{ }^{5}$ who found higher concentrations of $\mathrm{P}$ selectin in critically ill adults with sepsis. The lack of an increase in $\mathrm{P}$ selectin concentrations contrasts with the rise seen in shed E selectin and ICAM-1. Both E selectin and ICAM-1 depend on de novo protein synthesis and so have a slower time course than $\mathrm{P}$ selectin, which is released from preformed stores. P selectin is also released from platelets. We found higher concentrations of $P$ selectin in those with higher platelet counts. This relation was not found by Sakamaki et al. ${ }^{5}$

It is curious that there was no relation between the concentrations of TNF or LOS, both of which are potent stimulators of endothelial cell activation in vitro, and the measured concentrations of shed endothelial cell adhesion molecules. The cytokines and adhesion molecules were measured in samples taken at the same time, although the response of the endothelial cell to activation will occur some time after exposure to LOS or TNF. On these grounds, one would expect a better rela- tion between $\mathrm{P}$ selectin and the cytokines, and indeed the only relation found was one between IL-10 and P selectin, where higher concentrations of IL-10 were found in children with lower concentrations of $\mathrm{P}$ selectin.

In summary, we have demonstrated a relation between meningococcal disease and the release of cell adhesion molecules by activated endothelial cells. E selectin is higher in children with meningococcal disease than controls, and higher still in those with severe disease. Those who subsequently died did not have the highest values. The concentrations of ICAM-1 are higher in children with meningococcal disease. P selectin concentrations were not higher in children with meningococcal disease, and indeed those who died had lower values.

This work was supported by the Johanne Holly meningitis research fund.

1 Frenette PS, Wagner DD. Adhesion molecules-part II: blood vessels and blood cells. N Engl f Med 1996;335:435 .

2 Brandtzaeg P, Kierulf P, Gaustad P, et al. Plasma endotoxin levels as a predictor of multiple organ failure and death in systemic meningococcal disease. F Infect Dis 1989;159:195systernic
204.
Waage

3 Waage A, Halstensen A, Espevik T. Association between tumor necrosis factor in serum and fatal outcome in patients with meningococcal disease. Lancet 1987;i:355-7.

Girardin E, Grau GE, Dyer J-M, Roux-Lombard P, the J5 Study Group, Lambert P-H. Tumor necrosis factor and interleukin in the serum of children severe infectious purpura. N Engl f Med 1988;319:397-400.

Sakamaki F, Ishizaka A, Handa $M$, et al. Soluble form of P-selectin in plasma is elevated in acute lung injury. Am $\mathcal{F}$ Respir Crit Care Med 1995;151:1821-6.

6 Seth R, Raymond FD, Makgoba WM. Circulating ICAM-1 isoforms: diagnostic prospects for inflammatory and immune disorders. Lancet 1991;338:83-4

7 Cowley HC, Heney D, Gearing AJH, Hemingway I, Webster NR. Increased circulating adhesion molecule concentrations in patients with the systemic inflammatory response syndrome: a prospective cohort study. Crit Care Med 1994; 22:651-7.

8 Sessler CN, Windsor AC, Schwartz M, et al. Circulating ICAM-1 is increased in septic shock. Am f Respir Crit Care Med 1995;151:1420-7.

9 Newman W, Beall LD, Carson CW, et al. Soluble E-selectin is found in supernatants of activated endothelial cells and is elevated in the serum of patients with septic shock. $\mathcal{f}$ Immunol in the serum of

10 Klein NJ, Levin M, Strobel S, Finn A. Degradation of glycosaminoglycans and fibronectin on endotoxin-stimulated endothelium by adherent neutrophils: relationship o CD11b/CD18 and L-selectin. F Infect Dis 1993;167: $890-8$

11 Friedman G, Jankowski S, Shahla M, et al. Administration of an antibody to E-selectin in patients with septic shock. Crit Care Med 1996;24:229-33.

2 Ridings PC, Windsor ACJ, Jutila MA, et al. A dual-binding antibody to E- and L-selectin attenuates sepsis-induced lung injury. Am $\mathcal{F}$ Respir Crit Care Med 1995;152;247-53

13 Tan TQ, Smith CW, Hawkins EP, Mason EO, Kaplan SL. Hematogenous bacterial meningitis in an intracellular adhesion molecule-1 deficient infant mouse model. F Infect Dis 1995;171:342-9.

14 Boldt J, Mueller M, Heesen M, Neumann K, Hempelmann $\mathrm{GG}$. Influence of different volume therapies and pentoxifylline infusion on circulating soluble adhesion molecules in critically ill patients. Crit Care Med 1996;24:385-91.

15 Fortenberry JD, Huber AR, Owens ML. Inotropes inhibit Fortenberry JD, Huber AR, Owens ML. Inotropes inhibit
endothelial cell surface adhesion molecules induced by endothelial cell surface adhesion molecules
interleukin-1 $\beta$. Crit Care Med 1997;25:303-8.

16 Bruserud O, Aksela PE, Bergheim, J, Nesthus I. Serum concentrations of E-selectin, P-selectin, ICAM-1 and interleukin 6 in acute leukaemia patients with chemotherapyinduced leucopenia and bacterial infections. $\mathrm{Br} F$ Hematology 1995;91:394-402.

17 Jakobsen PH, Morris-Jones S, Ronn A, et al. Increased plasma concentrations of sICAM-1, sVCAM-1 and sELAM-1 in patents with Plasmodium falciparum or $\mathrm{P}$ vivax malaria and association with disease severity. Immunology 1994;83:665-9

18 Sinclair JF, Skeoch CH, Hallworth D. Prognosis of meningococcal septicaemia. Lancet 1987;ii:39.

19 Marzouk O. Clinical and laboratory aspects of meningococcal disease in children. MD Thesis, 1994. University of Liverpool.

20 Riordan FAI, Marzouk O, Thompson APJ, Sills JA, Hart CA. Proinflammatory and anti-inflammatory cytokines in meningococcal disease. Arch Dis Child 1996;75:453-4. 